\title{
Benefit-Risk of Immunomodulators in Multiple Sclerosis. Alemtuzumab and Next?
}

\section{Francois Curtin*}

GeNeuro SA, Geneva, Switzerland

\begin{abstract}
End of December 2013, alemtuzumab, a monoclonal antibody for multiple sclerosis (MS) with an immunomodulator action targeting CD52 receptors and inducing decrease counts of lymphocytes B and T was rejected by the Food and Drug Administration, as the registration dossier had not shown convincing evidence that the benefit of the drug would overreach its safety risks. The question is in fact more global as immunomodulators constitute the majority of the newly registered drugs and drugs in clinical development for MS. The knowledge accumulated by the time these drugs are registered does not suffice to ascertain their safety profile. This is preoccupying as these treatments are intended to be administered to young patients who need to be treated for decades. Cumulative risks over years with such treatments are at the moment unknown but are certainly non negligible. Despite significant benefits of immunomodulation in the treatment of MS, the solution for treatment of disorders with auto-immune origin is likely to come from targeting more specific factors to avoid long-term depression of the immune system. Research and development in this direction need to be actively encouraged.
\end{abstract}

Keywords: Multiple sclerosis; Immunomodulation; Immunosuppression; Safety risk; Benefit-risk ratio

By the 30th of December 2013, the Boston-based company Genzyme, part of the Sanofi Pharmaceutical group, announced that it had received a Complete Response Letter from the U.S. Food and Drug Administration (FDA) for its supplemental Biologics License Application seeking approval of alemtuzumab (Lemtrada ${ }^{\circledR}$ ) for the treatment of relapsing forms of multiple sclerosis (MS). A Complete Response Letter informs companies that an application is not ready for approval. The FDA wanted to see more evidence from controlled clinical trials that demonstrate the benefits of alemtuzumab outweigh the safety risk of the drugs [1]. Alemtuzumab, recently approved in the European Union, Canada and Australia, is a monoclonal antibody that selectively targets the CD52 receptors expressed on the surface of $\mathrm{B}$ and $\mathrm{T}$ cells: this induces a depletion of lymphocytes over the long term. Alemtuzumab was originally developed to treat B-cell chronic lymphocytic leukemia. The mode of administration is by intravenous infusion with treatment cycles of a few days: in the pivotal Phase III studies CARE-MS I and II, the first cycle was of 5 days during which patients received 5 infusions of $12 \mathrm{mg}$ of alemtuzumab, after one year, the second treatment cycle lasted 3 days with 3 IV infusions of $12 \mathrm{mg}$ alemtuzumab. The efficacy of the drug appears high as in the first Phase III study (CARE MS I) [2] including 563 patients with relapsing remitting MS (RRMS), alemtuzumab was compared to interferon $\beta 1 \mathrm{a}$, a reference MS treatment; the annualized relapse rate ratio (ARR), a standard metrics for clinical trials in RRMS, was of 0.18 in the alemtuzumab arm representing a decrease of 53\% compared to interferon $\beta 1$ a. In the other Phase III trial (CARE-MS II) [3] in 798 RRMS patients, the ARR was 0.26 in the alemtuzumab $12 \mathrm{mg}$ group, i.e. a decrease of $50 \%$ compared to the group treated with interferon $\beta 1$ a. These efficacy results appear all the more significant as differences of ARR of similar magnitude were seen with recently registered MS drugs such as fingolimod or dimethyl fumarate, but in comparison to placebo and not to an active MS treatment. The safety profile of alemtuzumab includes very frequent infusion reactions (headache, rash, nausea, pyrexia, etc), a tendency to more frequent infections (upper respiratory, urinary and herpetic infections in particular), and the occurrence of B cell mediated autoimmune disorders such as thyroid disorders (hyper- or hypothyroidism), immune mediated thrombocytopenia and anti-basal membrane antibody associated glomerulonephritis [4]. Lymphocyte counts decrease after each treatment cycle with a return of $\mathrm{B}$ cells to normal values in about 6 months while $\mathrm{T}$ cell counts in peripheral blood stay low for 12 or more months [5]. The long lasting lymphopenia represents a safety risk for patients treated with alemtuzumab. This risk was considered as too high to convince the FDA for an approval.

The safety issue raised by the FDA extends beyond alemtuzumab and actually concerns the benefit-risk of the whole class of immunomodulators/immunosuppressors which are now proposed for the treatment of RRMS and at least for certain of them such as fingolimod, natalizumab, ocrelizumab or siponimod are tested in progressive forms of MS. Not surprisingly, the European Medicine Agency (EMA) in its revised draft guidelines for MS treatments [6] has specifically highlighted the issue of the benefit-risk of the class of drugs. MS is an inflammatory and demyelinating, neurodegenerative disorder of the central nervous system (CNS) with an auto-immune mechanism, although the etiology remains unknown [7]. So far, immunomodulation - often an euphemism for immunosuppression has been the therapeutic mainstream for the treatments of MS, actually the sole treatment of the RRMS form, as for the progressive forms of the disease, there are almost no registered drugs [8]. Immunomodulators are efficacious in reducing the frequency of relapses in RRMS patients as well as for decreasing the progression of the disease at least over the short term. The first drugs developed for MS were beta-interferon and glatiramer acetate. The exact mechanism of action of these

*Corresponding author: Francois Curtin, GeNeuro SA Chemin des Aulx 18 CH-1228 Plan-les-Ouates/Geneva, Switzerland, Tel: +41 2279450 85; E-mail: fc@geneuro.com

Received January 22, 2014; Accepted February 20, 2014; Published February 25, 2014

Citation: Curtin F (2014) Benefit-Risk of Immunomodulators in Multiple Sclerosis Alemtuzumab and Next? J Neurol Neurophysiol 5: 197. doi:10.4172/21559562.1000197

Copyright: (c) 2014 Curtin F. This is an open-access article distributed under the terms of the Creative Commons Attribution License, which permits unrestricted use, distribution, and reproduction in any medium, provided the original author and source are credited. 
drugs still remains not fully understood, however their action on the immune system is sufficiently moderate not to increase the risk of infections or cancers $[9,10]$. Following these two drugs, much stronger immunomodulators were developed, the first being natalizumab followed by fingolimod and more recently teriflunomide and fumarate. These treatments have direct impact on the lymphocyte response, circulation or penetration in the tissues. This also true for the MS drugs currently in development: the clinical development pipeline in MS is constituted in majority of drugs which are impacting on different components of the immune system: in Phase II and III, 13 out of 16 products are acting on the immune system as the principal mode of action [8].

Several of the immunomodulator/immunosuppressor molecules in MS were originally developed for conditions where a very strong control of the immune system was deemed necessary to prevent a lethal evolution of these disorders, as in case of graft rejection for transplantation (fingolimod) or for lymphocytic leukemia (alemtuzumab, rituximab, etc...). Risks of immunomodulation/ immunosuppression include the occurrence of infections by opportunistic agents [11]. Among those, viruses such as herpesviridae (VZV, CMV, EBV, HSV...) are frequently seen as well as viruses such as the polyoma JC virus responsible for progressive multifocal leukoencephalopathy (PML). This serious disorder is observed during natalizumab treatment and, recently, cases have been reported in association with the new MS immunemodulators [8]. Among bacterial infections, those caused by Mycobacterium tuberculosis, Listeria monocytogenes, Salmonella, Pneumocystis jirovecii are typical. The safety risk concerns also cancers: viral-associated cancers such as lymphoma, skin cancers, cancer of the lips, Kaposi sarcoma, hepatocellular carcinoma, cervical cancer are more prevalent with drugs inducing immunosuppression [11]. Also certain of these drugs can induce a paradoxical activation of the immune cells such as T, B or NK cells with the risk of inducing auto-immune disorders: this was already observed with alemtuzumab as mentioned before as well as with anti-TNFa biologics [12].

The difficulty in the benefit:risk assessment of these drugs is to properly assess the immunosuppression risks over a clinical development including only a limited number of patients: for example alemtuzumab included 1486 patients, equivalent to more than 5,400 patient-years of exposure [13] whereas, for example, fingolimod included 2615 patients with 4582 patient-years of exposure [14]. The Haenszel rule of 3 states that if you don't see a given adverse event in a population of $\mathrm{n}$ patients, there is $95 \%$ chance that the highest incidence of the adverse event is $3 / \mathrm{n}$ [15]. Therefore, let's say, if one does not see a particular opportunistic infection in a clinical development program of 5,000 patient-years, the highest incidence rate of this infection will be $3 / 5,000=0.6$ case per 1,000 patients per year, which is relatively rare. For example, the incidence of progressive multifocal leukoencephalopathy (PML) in clinical trials with natalizumab was estimated at 1 case per 1000 patients in clinical trials [16]: assuming an average duration of 2 years for Phase III studies in MS, this suggests an incidence rate of 0.5 per 1,000 patients per year. Therefore a clinical development can fail to detect series of clinically significant adverse drug reactions (ADR) simply because the number of patients and the length of follow-up are limited at the time of registration.

MS is a chronic disease starting in young adults; the probability to get $\mathrm{ADR}$, even rare, over a long duration of treatment becomes higher due to long-term exposure. Assuming that the median time for a RRMS patient to convert to secondary progressive MS is of 19 years [17] and that a RRMS patient is treated with an immunosuppressor as long as she or he suffers from RRMS, the probability to get at least one occurrence of the given opportunistic infection with the incidence rate mentioned above during this period is the opposite probability of the probability not to get the infection during the 19 year period, i.e. 1 - (1 - 0.0006) $19=0.011$, a probability of $1.1 \%$, which makes this ADR much more frequent and problematic.

For ADR with a delayed onset, the issue comes from the length of follow-up: the insufficiency of the clinical development is not due to sample size but rather to duration. For cancers, the onset of virusassociated cancers as seen after immunosuppression takes a few years: the median time of onset for cancers associated with immunosuppression after transplantation is about 5 years [18]. Therefore the risk of lymphoma which may occur with an immunosuppressor will be observed only after a couple of years of post-marketing surveillance [18]. A similar comment applies to auto-immune conditions following alemtuzumab: this was studied in 248 alemtuzumab patients and autoimmune disorders (Graves disease, ITP and Goodpasture syndrome) observed in 55 of these patients developed with a median time of 41 months [4].

The evaluation of drug risk and the assessment of the benefit:risk ratio is a dynamic process and this assessment will continue to evolve during the whole lifecycle of the product [19]: the immunosuppression risk and the benefit:risk ratio of drugs based on this mode of action is certainly not established at the time of registration. This requests an assessment over the long-term.

\section{Conclusion}

Immunomodulation allowed unquestionable progresses in the therapeutic of MS disease by significantly decreasing MS relapses for patients with RRMS, and immunomodulators appear to positively influence the disease evolution [20], although some long-term retrospective study failed to confirm this observation [21]. However, the safety risk profile associated with this class of medications makes their prescription and monitoring a difficult task. Individual risk assessment of patients, careful considerations of contra-indications, such as JC virus immunity status or concomitant treatment with other immunomodulators, and expertise in the administration of these drugs are necessary steps to optimize the treatment with this therapeutic class [11]. As the degenerative process which leads to progression of disability and neurodegeneration in MS may evolve independently of the active immunological process [7] and that the long term impact of immunomodulation/immunosuppression on neurodegeneration still need to be ascertained, immunosuppression is probably not the definitive answer to MS. Alternative therapeutic solutions have to be developed and proposed in the therapeutic armamentarium. An interesting alternative could come from drugs which intend to neutralize specific pathogenic mechanisms appearing associated with MS, without affecting the immune system. Some drugs acting on such factors are now in early clinical development, among them a couple of monoclonal antibodies (mAb): BIIB-033 neutralises the LINGO-1 protein, an inhibitor of oligodendrocyte differentiation [22], GNbAC1 neutralises the MSRV-Env, a pro-inflammatory protein inhibitor of oligodendrocyte differentiation [23], VX15 blocks Semaphorin 4D impacting on remyelination capacity and inflammation [24]. These mAbs targeting specific pathogenic factors may open the way to more specific treatments with a potential better tolerance especially over the long term. However, these treatments have not passed the proof of concept stage and further clinical studies need to be performed.

In general, the benefit-risk of treatments for autoimmune disorders such as MS will have to be reconsidered in the future and the need 
Citation: Curtin F (2014) Benefit-Risk of Immunomodulators in Multiple Sclerosis. Alemtuzumab and Next? J Neurol Neurophysiol 5: 197. doi:10.4172/2155-9562.1000197

to a better understanding of the causal factors in these disorders is a necessary step to improve their therapeutics.

\section{References}

1. http://news.genzyme.com/press-release/genzyme-receives-completeresponse-letter-fda-lemtrada-alemtuzumab-application

2. Cohen JA, Coles AJ, Arnold DL, Confavreux C, Fox EJ, et al. (2012) Alemtuzumab versus interferon beta $1 \mathrm{a}$ as first-line treatment for patients with relapsing-remitting multiple sclerosis: a randomised controlled phase 3 trial. Lancet 380: 1819-1828.

3. Coles AJ, Twyman CL, Arnold DL, Cohen JA, Confavreux C, et al. (2012) Alemtuzumab for patients with relapsing multiple sclerosis after diseasemodifying therapy: a randomised controlled phase 3 trial. Lancet 380: 18291839.

4. Costelloe L, Jones J, Coles A (2012) Secondary autoimmune diseases following alemtuzumab therapy for multiple sclerosis. Expert Rev Neurother 12: 335-341.

5. Cross AH, Klein RS (2013) Comment: assessing humoral immunocompetence after alemtuzumab treatment in MS. Neurology 81: 875.

6. Vinhas de Souza M, Keller-Stanislawski B, Blake K, Hidalgo-Simon A, Arlett P, et al. (2012) Drug-induced PML: a global agenda for a global challenge. Clin Pharmacol Ther 91: 747-750.

7. McFarland HF, Martin R (2007) Multiple sclerosis: a complicated picture of autoimmunity. Nat Immunol 8: 913-919.

8. Curtin F, Hartung HP (2014) Novel therapeutic options for multiple sclerosis. Expert Rev Clin Pharmacol 7: 91-104.

9. Sandberg-Wollheim M, Kornmann G, Bischof D, Moraga MS, Hennessy B et al. (2011) The risk of malignancy is not increased in patients with multiple sclerosis treated with subcutaneous interferon beta-la: analysis of data from clinical trial and post-marketing surveillance settings. Mult Scler 17: 431-440.

10. Achiron A, Barak Y, Gail M, Mandel M, Pee D, et al. (2005) Cancer incidence in multiple sclerosis and effects of immunomodulatory treatments. Breast Cancer Res Treat 89: 265-270.

11. Riminton DS, Hartung HP, Reddel SW (2011) Managing the risks of immunosuppression. Curr Opin Neurol 24: 217-223.

12. Brennan FR, Morton LD, Spindeldreher S, Kiessling A, Allenspach R, et al. (2010) Safety and immunotoxicity assessment of immunomodulatory monoclonal antibodies. MAbs 2: 233-255.
13. http://www fda gov/downloads/AdvisoryCommittees/CommitteesMeetingMaterials/Drugs/PeripheralandCentralNervousSystemDrugsAdvisoryCommittee/ UCM374188.pdf.

14. http://www.fda.gov/downloads/Advisory\%20Committees/CommitteesMeetingMaterials/Drugs/Peripheraland\%20CentralNervousSystemDrugsAdvisoryCommittee/UCM214675.pdf.

15. Haenszel W, Loveland DB, Sirken MG (1962) Lung-cancer mortality as related to residence and smoking histories. I. White males. J Natl Cancer Inst 28: 947 1001.

16. Bloomgren G, Richman S, Hotermans C, Subramanyam M, Goelz S, et al. (2012) Risk of natalizumab-associated progressive multifocal leukoencephalopathy. N Engl J Med 366: 1870-1880.

17. Confavreux C, Vukusic $S$ (2006) Natural history of multiple sclerosis: a unifying concept. Brain 129: 606-616.

18. Wasson S, Zafar MN, Best J, Reddy HK (2006) Post-transplantation lymphoproliferative disorder in heart and kidney transplant patients: a singlecenter experience. J Cardiovasc Pharmacol Ther 11: 77-83.

19. Curtin F, Schulz $P$ (2011) Assessing the benefit: risk ratio of a drug--randomized and naturalistic evidence. Dialogues Clin Neurosci 13: 183-190.

20. Degenhardt A, Ramagopalan SV, Scalfari A, Ebers GC (2009) Clinica prognostic factors in multiple sclerosis: a natural history review. Nat Rev Neuro 5: $672-682$.

21. Shirani A, Zhao Y, Karim ME, Evans C, Kingwell E, et al. (2012) Association between use of interferon beta and progression of disability in patients with relapsing-remitting multiple sclerosis. JAMA 308: 247-256.

22. Tran J, Palaparthy R, Brosofsky K, Ray S, Rana J, et al. (2012) Safety tolerability and pharmacokinetics of the anti-LINGO-1 monoclonal antibody BIIB033 in healthy volunteers and subjects with multiple sclerosis. Presented at: 63 rd Annual Meeting of the American Academy of Neurology; New Orleans LA, USA.

23. Curtin F, Lang AB, Perron $H$, Laumonier M, Vidal V et al. (2012) GNbAC1, a humanized monoclonal antibody against the envelope protein of multiple sclerosis-associated endogenous retrovirus: a first-in-humans randomized clinical study. Clin Ther 34: 2268-2278.

24. Fisher T, Reilly C, Winter L, Veerararaghavan A, Jonason J et al. (2012) Development of anti-SEMA4D monoclonal antibody for the treatment of multiple sclerosis. Presented at: 28th Congress of the European Committee for Treatment and Research in Multiple Sclerosis Lyon, France.
This article was originally published in a special issue, Neurodegenerative Diseases: Symptoms and Therapeutics handled by Editor(s). Dr. Jin J Luo, Temple University School of Medicine, USA 